\title{
The problematic symmetry between brain birth and brain death
}

\author{
D Gareth Jones University of Otago, Dunedin, New Zealand
}

\begin{abstract}
The possible symmetry between the concepts of brain death and brain birth (life) is explored. Since the symmetry argument has tended to overlook the most appropriate definition of brain death, the fundamental concepts of whole brain death and higher brain death are assessed. In this way, a context is provided for a discussion of brain birth. Different writers have placed brain birth at numerous points: 25-40 days, eight weeks, 22-24 weeks, and 32-36 weeks gestation. For others, the concept itself is open to question. Apart from this, it needs to be asked whether a unitary concept is an oversimplification. The merits of defining two stages of brain birth, to parallel the two definitions of brain death, are discussed. An attempt is then made to map these various stages of brain birth and brain death onto a developmental continuum. Although the results hold biological interest, their ethical significance is less evident. Development and degeneration are not interchangeable, and definitions of death apply specifically to those who are dying, not those who are developing. I conclude that while a dual concept of brain death has proved helpful, a dual concept of brain birth still has problems, and the underlying concept of brain birth itself continues to be elusive.

(Fournal of Medical Ethics 1998;24:237-242)
\end{abstract}

Keywords: Brain birth (life); brain death; whole brain death; higher brain death; developmental continuum

\section{Introduction}

In the 30 years since the designation of brain death as a new criterion for death, attention has been directed towards the central role of the nervous system in a number of areas of ethical decision-making. The notion that there exists a neurological end-point to human life has led to efforts at defining a neurological starting-point. This latter quest has led to the concept of brain birth (or brain life), signifying the converse of brain death. ${ }^{1-3}$ Nevertheless, many scientific and ethical queries remained. ${ }^{45}$

The quest for a neurological marker of the first events of human life owes its impetus to the perceived symmetry between processes at the beginning and end of life. Burgess and Tawia write: "If conscious experiences ... are the aspect of our lives we value when we look forward, considerations of symmetry dictate that we first acquire a capacity for what we most value in our lives when we first become conscious". ${ }^{5}$ They view the beginning of consciousness as the beginning of "cortical life". A concrete expression of this trend has been provided by Sass, who advocates the legal protection of "personal life (animate life) from the beginning of brain functioning (brain life) to its end (brain death)". ${ }^{\circ}$

Yet brain birth describes a progressive phenomenon which is leading somewhere new, whereas brain death describes the final point of an existence. ${ }^{78}$ The contrast between these two states is striking, with brain birth centring around a discovery concerning personhood and brain death around a definition, ${ }^{7}$ and with the order of neural embryogenesis standing against the disorder of neural death. ${ }^{49}$ The symmetry argument also simplifies brain death criteria by overlooking the most appropriate definition of brain death, either loss of function of the whole brain (destruction of the cerebral hemispheres plus brain stem, or brain stem alone since this is a precursor of whole brain death), or irreversible loss of higher brain functions (total loss of consciousness and awareness, loss of cognitive faculties, representing widespread destruction of the cerebral hemispheres). This is a distinction between a vitalist interpretation, with its emphasis on biological integration, and a personalist interpretation, stemming from the significance of sentience or consciousness for the existence of persons. The contrast is between "mere human biological life" and "being alive as a person". ${ }^{10}$

Unfortunately, brain death has generally provided an undefined context for discussions regarding how best to describe events at the beginning of life. For instance, it is not clear what definition of brain death is being used in symmetry-based arguments. This gap is a far more serious one than generally realized, and the debate on brain birth can be taken much further by exploring its dimensions alongside those of brain death. 


\section{Brain death - fundamental concepts}

WHOLE BRAIN DEFINITION

The Harvard criteria for a permanently nonfunctioning brain pointed to total and irreversible loss of functioning of the whole brain. ${ }^{11}$ A flat electroencephalogram (EEG) was recommended as a confirmatory test.

Whole brain death refers to the irreversible cessation of function of both the brain stem and higher parts of the brain, including the cerebral hemispheres, although in the United Kingdom it refers to the brain stem alone, on the assumption that loss of brain stem functions is rapidly followed by cessation of function of the higher parts of the brain. In other words, brain stem death is seen as synonymous with death of the individual, since loss of functions associated with the brain stem results in the individual ceasing to function as an independent biological unit. ${ }^{12}$

From this it follows that death does not occur until both the brain as a whole and the body as a whole are irreversibly dysfunctional. This is a biological concept, and death is recognized as having taken place without any reference to a capacity for self-consciousness or personhood. However, in spite of the brain as a whole being considered dead, isolated functions may continue within the brain, and cellular activity in localized regions of the brain may also remain intact. ${ }^{13} 14$

Although proponents of a whole brain definition allegedly use the entire brain as their criterion of death, the identification of a higher brain function, such as sentience, tends to emerge as an essential feature. For some, a whole brain definition is an unstable compromise between the deaths of persons and organisms. ${ }^{15}$ It has to be asked whether individuals with intact integrative functions (signifying an intact brain stem), but without the marks of personhood (damage to the cerebral hemispheres) are alive in any meaningful sense. Some writers do not think so, arguing that the life that continues after destruction of the cerebral hemispheres (neocortex) is no more than metabolic activity, equivalent to that of a disembodied human organ or cell surviving in vitro. ${ }^{16}$

\section{HIGHER BRAIN DEFINITION}

A higher brain definition refers to destruction of the cerebral hemispheres alone, with retention of brain stem function. A move in this direction is a move away from utilizing criteria shared with other animals to a definition focusing on functions characteristic only of humans. Interest centres on the irreversible loss of higher brain functions, such as the capacity for consciousness, and the capacity for remembering, judging, reasoning, acting, enjoying and worrying. If these are regarded as characteristic of human existence and of \&ur meaning as human persons, they may be regarded as the sole functions of ultimate importance when defining brain death. ${ }^{17}$ What counts as a living human being is the presence of the capacity both organic and mental functioning, rather than the persistence of isolated brain stem reflexes lowing destruction of the cerebral hemisphers. The centrality of the person lies at the heart of $\ln _{\mathrm{e}}$ higher brain definition, and this in turn, focuses attention on our humanness and on those responsibilities lying at the core of human community.

COMPLEXITY OF BRAIN DEATH

These definitions of brain death highlight complexity of death, the variety of factors to considered, and the range of neurobiological and philosophical components to be taken ig ifo account. $^{18}$

For many people, there is a difference betwe exn death of the body and death of the brain (regandless of the definition employed). However, bodily life is still life, but is this meaningful life iro a human sense? It is tempting to conclude that he meaningfulness of an individual's existenge e $_{\infty}$ is open to question once irreversible higher Brajn death is definitely diagnosed: that individual fife is at an end, the irreversibility of the conftion eliminating any potential for future relationships, for self-awareness, or for plans of any description. On the other hand, the opposite perspective succinctly expressed by Lamb: "Life without conscious experience may be meaningless, possibly futile, but it does not amount to death". ${ }^{19}$

These considerations are relevant for those of brain birth. When can an embryo/fetus be saidoto be alive in any meaningful sense? Is it at the físt glimmerings of a nervous system (neural plate/ neural tube), at the first signs of the potential for consciousness/sentience (cerebral hemispheres recognizable/initial manifestation of localized EEG activity), or when there is sufficient strifctural organization for the nervous system to function in a coordinated manner (EEG activity ch acterized by little electrocerebral silence)? I\$ it possible to translate perspectives on brain defith directly into considerations of brain birth? If this is done, different characteristics will emerge ${ }_{\text {as }}$ relevant.

\section{Brain birth}

The concept of brain birth has featured $\vec{D}_{\text {in }}$ attempts to elucidate a moral point- of demarcation, prior to which experimental prosedures may be undertaken on human embryos but after which they are forbidden. ${ }^{60}$ Numerous 
writers have attempted to pinpoint what they regard as the biological substratum for personal life, ${ }^{51-23}$ with attention on development of the cerebral cortex and on identifying the first moments of conscious experience. Some have set this at 25-40 days gestation. ${ }^{24}$ For others, eight weeks gestation represents the point at which the brain is capable of consciousness. From this point onwards, the "biography" of the individual has begun $^{25}$; alternatively, this level of brain activity signifies the emergence of a person, ${ }^{22}$ makes possible an holistic level of life, ${ }^{21}$ leads to affective recognisability by other people,${ }^{26}$ or denotes the beginnings of sentience. ${ }^{27} 28$

Goldenring, ${ }^{2}$ who originally proposed the "brain-life theory", based his view on evidence that the subcortical brain comes into being as an organized unit at five weeks, with cerebral hemispheres differentiating at seven weeks, and EEG activity commencing at eight weeks. According to the brain-life theory, a human being is alive whenever a functioning human brain is present. Thus, the commencement of EEG activity is seen as the starting point for human (personal) existence, since it marks integration of the brain as a whole. ${ }^{3}$ The attractiveness of this theory stems from an apparent symmetry between the beginning and end of human existence, using the converse of brain death as a model for thinking about the initiation of coordinated neural activity.

Others have sought a much later beginning. Gertler $^{20}$ proposed 22-24 weeks gestation, on the basis that the neocortex begins producing EEG waves at this time. Underlying this proposal is the view that human cognition is the beginning of cognitive capability and the point at which protection of personhood should begin. In similar fashion, Burgess and Tawia ${ }^{5}$ defined a functioning brain as one where there is identifiable activity of the kind that normal adult brains (cortices) indulge in. They argue that what is required is a critical minimum level of structural organisation, with functional components present and mature enough to perform. On the basis of EEG readings, they conclude that a fetus becomes conscious at 32-36 weeks gestation.

The significance of EEG activity for this discussion is unmistakable. The earliest very localized EEG activity appears at ten weeks, ${ }^{29}$ with more generalized activity at $22-23$ weeks gestation. This activity, however, is discontinuous, with long periods of electrocerebral silence, a characteristic of the immature nervous system. ${ }^{30}$ Premature infants with gestational ages less than eight months have long periods during which the EEG shows no activity. Gradual changes in EEG characteristics occur over the first few years of postna- tal life, until adult patterns predominate around age seven or eight years. ${ }^{9}$

Also relevant here is the issue of fetal awareness, which has been placed at not earlier than 26 weeks gestation by a 1997 working party of the Royal College of Obstetricians and Gynaecologists. ${ }^{32}$ According to this working party, this is the minimum stage of structural development necessary to confer awareness upon the developing fetus, with structural integration of peripheral nerves, spinal cord, brain stem, thalamus, and cerebral cortex.

In view of the above, can any one notion of brain birth encapsulate the state of development or maturity over a 30-week period? This represents 75 per cent of gestation, from early in the embryonic period to late in the fetal period. Such a vast time period is too crude to prove convincing embryologically, and is too diffuse to prove helpful ethically.

\section{CRITIQUE OF BRAIN BIRTH}

Apart from this consideration, the brain birth concept is not universally accepted. For Moussa and Shannon, ${ }^{7}$ it is neither a defensible nor useful notion, since it is a metaphysical concept. The dependence placed on the physical maturity of one bodily system implies that metaphysical status can be inferred from scientific data - a notion that Moussa and Shannon strongly reject. For them, personhood is a social and moral construct and, they contend, biological realities neither guarantee the presence of, nor constitute the definition of, a person. According to these authors, a functioning nervous system is a presupposition only of physical activity, with an integrated nervous system being required for intellectual activity. As others have suggested, scientific criteria have a role in helping direct our moral gaze, demonstrating when a nervous system exists with sufficient material complexity to embody those capacities judged morally pertinent. ${ }^{33}$ The tenor of these sentiments points towards a definition of brain birth modelled on a higher brain definition of death - if such a definition is even contemplated at the beginning of life.

In terms of scientific criteria, the dominant feature of the developing brain is the laying down of different systems at different times, and the coordination of these systems relatively late in development. ${ }^{4}$ Some developmental sequences can only begin once the preceding sequence has been completed, some sequences occur relatively late in fetal life, while others are not completed until after birth. Consequently, if brain birth is placed early, numerous phases of development will not even have been initiated. Hence, if the 
concept is to be used, it would seem preferable to place it at a time when most developmental sequences have started, ${ }^{4}$ once again pointing to a definition based on a higher brain definition of brain death.

\section{Is there more than one definition of brain birth?}

Apart from any problems with the concept of brain birth, a crucial question is whether a unitary concept is an oversimplification. If there are two definitions of brain death, are there two definitions of brain birth?

The whole brain definition of death refers to the loss of major brain regions, including the brain stem. Is there a parallel at the beginning of life? Employing the appearance of brain stem functioning as one's criterion, brain birth would be placed at around 6-8 weeks gestation. I shall refer to this as brain birth $I$, which is a vitalist interpretation, with its emphasis on biological integration and its stress on mere human biological life. In contrast, a second definition may be determined by the beginning of consciousness at 24-36 weeks gestation. This is brain birth II, which parallels the personalist overtones of the higher brain definition of death, with a sufficiently well-developed neural organization to serve as the substratum from which self-consciousness and personal life subsequently emerge.

Do these two clearly delineated definitions of brain birth promote an understanding of the significance of early developmental events? In a developmental sequence, brain birth I always precedes brain birth II, while in a degenerative process at the end of life a higher brain definition of death (cortical/cerebral death) may or may not precede whole brain (brain stem) death. This variability is unavoidable, since degenerative processes lack the inbuilt sequence of developmental milestones. One of the defining features of whole brain death in adults is a lack of consciousness and an inability to feel pain. Symmetry with the brain death concept suggests that brain birth I should demarcate the beginning of the period when the fetus begins to develop sensation and feel pain, but as yet, the answer to this problematic question remains unclear. ${ }^{34-36}$ Rudimentary sensation seems to occur long before the stage when the nervous system can keep the fetus alive, but until a greater understanding of this process can be achieved, there exist no specific developmental milestones that establish the moment when a fetus becomes brain-alive in this sense. ${ }^{37}$ Even brain birth II is surrounded by ambiguity, although neural integration and the potential for conscious- ness, as depicted by cerebral cortical develळ ment, point to a relatively mature nervous system.

The realization that two separate timings brain birth are feasible enlarges the horizons of the debate, but at present fails to solve fundamental queries at the neurobiological level. This is esen more pronounced for ethical questions, since are left with major uncertainty whether brain bifinh I and/or brain birth II have moral significan®e. Levels of uncertainty look set to increase whos serious debate is undertaken on the possible repercussions of techniques that stimulate nonembryonic cells into totipotency, provide them with primitive human nervous systems but fai allow the development of any additional "human" characteristics. What impact would such scientific work have on the notion that brain birth signifies moral value?

\section{A continuum from life to death}

If brain life is taken as a notional indicator of brain function, it is possible to use two pathways to trầce brain life. One pathway represents normal de opment, from fertilization, through fetal ayd embryonic stages, to the child/adult, at whish point brain life is at a maximum. The othef is a pathological one, working in reverse, and tracing various pathological states as they depart brain life at its maximum, with dementia and persistent vegetative state (PVS)/anencephôfy representing increasing decrements until deathof the individual occurs. When the various defsi tions of brain death are superimposed upon these pathways, possible parallels can be explowd between normal development on the one havid and pathological deviations from the normal on the other. In this way, it is possible to compare क्in a tentative fashion) the degrees of brain fife evidenced by those designated as being dead usông a whole brain definition in contrast to a higher brain definition, as well as by anencephalics and those in a PVS. Against this background, ald using what is known of brain death, the next sep is to ask when a brain can be said to come ifo existence.

Using a higher brain definition, the answer Res somewhere in the vicinity of 24-36 weeks (broin birth II). If this is the case, the parallelism postulated here raises the question whether की embryo/fetus at all stages prior to about 22 we gestation can be meaningfully referred to tas "brain dead". This is a possibility if a brain bipth concept based on a higher brain definition of de th is accepted. However, the terminology is confusigig, since it is difficult to appreciate how something ean be dead when it has never lived. In addition, the developing nervous system is developing into 
"something", whereas the adult brain that is now non-functioning previously was "something". The problems of lack of symmetry remain. A "preconscious" nervous system is fundamentally different from a "post-conscious" nervous system; the potential of the first differs significantly from the memories represented by the second.

An alternative is to adopt a whole brain definition of death as the model on which to base a definition of brain birth (brain birth I). Taking this approach, brain birth is placed at around 6-8 weeks gestation, when the nervous system is still at a relatively early stage of development, and the neuromuscular system is sufficiently developed for spontaneous fetal movements to occur. ${ }^{32}$ While this is feasible, such early neural development does not constitute a brain-like organization in biological terms.

Even brain birth I does not signify the first beginnings of the nervous system. This happens with the appearance of the neural plate (18 days gestation), and then more obviously with the slightly later appearance of the neural tube (closing around 27 days). These early stages in nervous system development do not generally feature in discussions of brain birth, although they may be utilized in connection with the significance of the primitive streak or even with the onset of consciousness.

In conclusion, although it is possible to place the two-week embryo, eight-week embryo, 36week fetus, term fetus, newborn infant and child on a continuum, it is doubtful whether the biological interest elicited by this is matched by ethical significance. Even if an eight-week embryo is considered to display less brain life than, say, an adult in a PVS or an adult with dementia, one cannot conclude from this that an embryo at this stage of development has more of the characteristics of brain death than does either of these adult groups. Definitions of death apply specifically to those who are dying, not to those who are developing. Development and degeneration are not interchangeable. The problems encountered with translating brain death into neural system development serve to highlight the questionable foundation on which the entire edifice of the brain life concept is constructed.

Definitions of death are not purely biological, but are informed by moral judgments. While their base is, in part, biological, the character of the definitions owes more to ethical, social, and religious values. This applies also to the beginning of human life: hence my suspicions regarding the validity of the brain birth concept. Its biological base is significantly more fragile than that of brain death, and, as a result, far more is demanded of its ethical and social underpinnings. Brain death is proving a helpful concept, despite the fact that its dimensions remain open to debate and interpretation. Brain birth, even in its dual form discussed here, continues to be elusive.

\section{Acknowledgement}

Thanks to Barbara Telfer and Robyn Harris for their many contributions to my thinking on this topic.

$D$ Gareth Fones, BSc, MBBS, DSc, FIBiol is Professor of Anatomy and Structural Biology, University of Otago. He has also served as Acting Director of the Bioethics Research Centre, University of Otago.

\section{References}

1 Brody B. The morality of abortion. In: Beauchamp TL, Walters $\mathrm{L}$, eds. Contemporary issues in bioethics. Belmont, CA: Wadsworth, 1982:240-50.

2 Goldenring JM. Development of the fetal brain[letter]. New England fournal of Medicine 1982;307:564.

3 Goldenring JM. The brain-life theory: towards a consistent biological definition of humanness. Fournal of Medical Ethics 1985;11:198-204.

4 Jones DG. Brain birth and personal identity. Fournal of Medical Ethics 1989;15:173-8.

5 Burgess JA, Tawia SA. When did you first begin to feel it? locating the beginning of human consciousness. Bioethics 1996; 10:1-26.

6 Sass H-M. The moral significance of brain-life criteria. In: Beller FK, Weir RF, eds. The beginning of human life. The Netherlands: Kluwer Academic Press, 1994:57-70.

7 Moussa M, Shannon TA. The search for the new pineal gland: brain life and personhood. Hastings Center Report 1992;22:30-7.

8 Moussa M, Shannon TA. Brain metaphysics[letter]. Hastings Center Report 1993;23:45-6.

9 Jones DG. Manufacturing humans. Leicester: Inter-Varsity Press, 1987.

10 Engelhardt HT. Some persons are humans, some humans are persons, and the world is what persons make of it. In: Spicker SF, Engelhardt HT, eds. Philosophical medical ethics: its nature and significance. Dordrecht, Holland: Reidel Publishing, 1975: 183-94.

11 Ad Hoc Committee of the Harvard Medical School to Examine the Definition of Brain Death. A definition of irreversible coma. Fournal of the American Medical Association 1968;205: 337-40.

12 Pallis C. Whole-brain death reconsidered - physiological facts and philosophy. Fournal of Medical Ethics 1983;9:32-7.

13 Bernat JL, Culver CM, Gert B. On the definition and criterion of death. Annals of Internal Medicine 1981;94:389-94.

14 Grigg MM, Kelly MK, Celesia GG, Ghobrial MW, Ross ER. Electroencephalographic activity after brain death. Archives of Neurology 1987;44: 948-54.

15 McMahan J. The metaphysics of brain death. Bioethics 1995;9: 91-126.

16 Bartlett ET, Youngner SJ. Human death and the destruction of the neocortex. In: Zaner RM, ed. Death: beyond whole-brain criteria. Dordrecht, Holland: Reidel Publishing Company, 1988: 199-215.

17 Veatch RM. The impending collapse of the whole-brain definition of death. Hastings Center Report 1993;23:18-24.

18 Hauerwas S. Religious concepts of brain death and associated problems. Annals of the New York Academy of Sciences 1978;315: 329-38.

19 Lamb D. Death, brain death and ethics. London: Croom Helm, 1985.

20 Gertler GB. Brain birth: a proposal for defining when a fetus is entitled to human life status. Southern California Law Review 1986;59:1061-78.

21 Shea MC. Embryonic life and human life. Fournal of Medical Ethics 1985;11:205-9.

22 Tauer CA. Personhood and human embryos and fetuses. Fournal of Medicine and Philosophy 1985;10:253-66. 
23 Beller FK, Zlatnik GP. The beginning of human life. fournal of Assisted Reproduction and Genetics 1995;12:477-83.

24 Board for Social Responsibility (Working Party on Human Fertilisation and Embryology). Personal origins. London: CIO Publishing, 1985.

25 Kushner T. Having life versus being alive. fournal of Medical Ethics 1984;10:5-8.

26 Grobstein C. From chance to purpose: an appraisal of external human fertilization. Reading, Mass: Addison-Wesley Publishing, 1981 .

27 Singer P, Wells D. The reproductive revolution: new ways of making babies. Oxford: Oxford University Press, 1984.

28 Kuhse H, Singer P. Abortion and contraception: the moral significance of fertilization. See reference 6: 145-61.

29 Bergström RM, Bergström L. Prenatal development of stretch reflex functions and brainstem activity in the human. Annales Chirurgiae et Gynaecologiae Fennicae 1963;52:1-21.

30 Anderson CM, Torres F, Faoro A. The EEG of the early premature. Electroencephalography and Clinical Neurophysiol@ 1985;60:95-105.

31 Tawia S. When is the capacity for sentience acquired dur human fetal development? The fournal of Maternal-Fetal Meackcine 1992;1:153-65.

32 Royal College of Obstetricians and Gynaecologists. Fetal awareness: report of a working party. London: RCOG Press, 1997.

33 Flower $M$. Neuromaturation and the moral status of human fetal life. In: Doerr E, Prescott J, eds. Abortion rights and fetal "personhood". Long Beach: Crestline Press, 1989:71-85.

34 Kuljis RO. Development of the human brain: the emergence $\overline{\bar{O}} \mathrm{f}$ the neural substrate for pain perception and consciofs experience. See reference 6:49-56.

35 Lloyd-Thomas AR, Fitzgerald M. Reflex responses do not nêessarily signify pain. British Medical fournal 1996;313:797-8ळ

36 Szawarski Z. Probably no pain in the absence of "self". Britioh Medical fournal 1996;313:796-7.

37 Maynard-Moody S. The dilemma of the fetus: fetal research, medical progress and moral politics. New York: St Martin's Press, 1995. 